\title{
New Directions in Mental Health Care Evaluation
}

\section{Report of a workshop* held on 3 July 1987 at the Institute of Psychiatry to discuss a proposed new unit and consortium}

\author{
IsaAC Marks, Professor of Experimental Psychopathology, JosePh Connolly, Consultant Psychiatrist, and \\ Matthus Muijen, Research Worker/Honorary Senior Registrar, Institute of Psychiatry and Bethlem-Maudsley Hospital, \\ London SE5
}

\section{Overview}

Mental health care evaluation is a priority area as mental health care services are changing rapidly, more than are most other medical services. Budgets are finite, and there is concern for value for money in meeting the needs of patients and families. A one-day workshop brought together leading figures in the USA and Europe to discuss how a unit and consortium might be established to give cohesion and catalyse evaluative research in this fragmented field in the UK. Sound scientific data are available which have not yet been widely disseminated or used in policy making. There are rich opportunities for cross-national projects. The USA has intriguing innovations in evaluative research in mental health, some on a large scale, and these are relevant to the UK.

The health care evaluation unit (HCEU) would consist of full-time staff doing research, teaching and consultation.

*The workshop and this report of it were helped by grants from the Nuffield Provincial Hospitals Trust and the Department of Health and Social Security.
The HCEU would link with interested parties funded from outside the unit to co-ordinate an HCE network (consortium) whose components would co-operate according to research needs. The unit and consortium would be interdisciplinary and liaise with professional, planning, administrative and government bodies and the media, keeping them informed of developments and learning what the current concerns are that deserve research.

Early concerns could include the evaluation of DHSSfunded research in community care, the evolution of quick but reliable indicators of clinical and economic outcome for quality assurance in routine clinics, the computerisation of those indicators and of non-clinical health care data to be made available nationally for researchers, and the establishment of an academic course in mental HCE. The unit and consortium could do research and development for agencies on a fee-paying basis and market evaluative methods to interested parties. It would have to address scientific peers, patients and their families, care-givers, policymakers and fund-givers. There is a large research agenda of relevance to them all, and careful focus will be essential.

\section{Review}

Living with Schizophrenia: An educational package designed by Jackie Ferris and Roma Byer; published by Northern Schizophrenia Fellowship and distributed by Newcastle upon Tyne Polytechnic Products, 1986. Price not quoted.

This "educational package" contains four audiocassettes, each lasting approximately 30 minutes, and a booklet of 11 unnumbered pages. The tapes take the form of long narratives by a small number of patients and an even smaller number of relatives, interspersed with comments by, one presumes, a psychiatrist (Dr Farquharson receives a credit on the booklet). The quality of the recording is generally good though there is sometimes some unnecessary background noise and variable recording levels. The package is well-produced although it is irritating that costs have not been saved by recording on both sides of the cassettes. The booklet is also glossily produced and looks as if it would last well. Most of it is a description of the various methods for using the tapes for education or "counselling", particularly for "intensive counselling" which may be directed to parents, brothers or sisters, or older children.

My initial reaction to the first tape was very favourable. The various people on the tapes present fairly varied life experiences, but are all articulate and describe the florid symptoms of schizophrenia, and their reactions to them, well. It would serve as a useful educational tape for medical students. The booklet summarises the florid symptoms described in a useful way, and mentions symptoms that have been omitted.

Unfortunately my positive response ebbed away as the tapes progressed. The fact that the commentator is never introduced, that he appears on the tape with no very definite separation from the other speakers, and that his contributions are obviously edited in from a much longer and, at times rambling, commentary becomes increasingly irritating. An over-emphasis on florid symptoms becomes 
apparent as the tapes progress, and the inaccurate description of negative symptoms in the booklet (catatonia, depression, physical deterioration and the inability to perform basic tasks are all included) points up the lack of importance attached to them on the tapes.

This becomes particularly a problem when the tapes switch from patients' to relatives' accounts, with tape 3 . The relatives all appear to start from the presumption that people with schizophrenia are mentally incompetent and that this is a permanent state of affairs which begins with a person's first illness. "He's like a baby", says one parent, and the psychiatrist's comment is "These reactions are completely normal, the shock, guilt ... and the grief at the loss of the child that you've known and the replacement by someone rather different". Later on a GP is quoted, approvingly ("it's better to be brutal"), as saying, "You can forget your 16 year old son. He's gone for ever. You've got to start to learn to know somebody else". The systematic infantilisation of the patient is reinforced by the fact that the problem symptoms that receive most attention are delusions and hallucinations, and that these are treated as incomprehensible "silly ideas".

Two types of advice are contained in the tape concerning the parents' adjustment to the illness, and management of the patient's behaviour. Parents are enjoined to share with other parents, and to find out as much information as possible. It is half-recognised that parents become depressed and anxious especially soon after the diagnosis- the couple who are introduced on tape 3 and talk throughout tape 4 approvingly quote what a friend (friend?) said to them, "Neil has an illness, but your problem is self-pity ... snap out of it"-but there is no discussion of why some parents should react so badly and the only other "treatment" considered is a regular visit from a community nurse.

This lack of sensitivity for personal feeling is even more apparent in the discussions about management. Although there is obvious concern for "the child" (all the relatives quoted are parents), there is no mention of "the child's" feelings until the very end of the last tape and a lot of discussion of the sort of behavioural problems that preoccupy people who manage institutions: bad eating habits, aggression, spending too much time in bed, and interfering with the television. Management techniques are also recognisably institutional. Drug treatment and limit-setting are the most commonly mentioned.
"You [the family] are as important as the sufferer", says the psychiatrist, but these tapes make one wonder whether this should be so as far as mental health professionals are concerned. Should the community psychiatric nurse be visiting the parents on a weekly basis, or the patient? Is it just as good to tell parents that, unless Neil stops standing in front of the television, he will have to move out as to try and get Neil to tell his parents why he objects to them watching or to suggest that Neil rents his own television? These questions are not raised in the last two tapes, perhaps because the "sufferers" are there described as if they are dying ("it's one long bereavement"). As every psychiatrist knows, no-one talks to the dying about feelings.

If the parents recorded on the last tapes had had an opportunity to listen to the achievements of the patients featured on the first two, they may have come to different conclusions about schizophrenia. Instead, the examples only served to underline again that expectations in schizophrenia can so easily be self-fulfilling. Treat someone with schizophrenia as if it really were a dementing illness, and they will behave as if mentally handicapped.

I would happily recommend the first two tapes for teaching families and professionals about schizophrenia. I could not recommend the last two tapes for this purpose. Their one use in my unit will, I think, be to illustrate that people need models of schizophrenia to hang onto, and that we need to develop better ones. Personally I think that emphasising the person with schizophrenia's difficulty in handling non-verbal cues of emotion and social interaction as the primary defect, and presenting psychosis as a second stage catastrophe response if uninterpretable cues become overwhelming, is a useful point at which to start. On this model, schizophrenia is more like aphasia than the dementia model implied in the tapes. I know which condition I'd rather other people assumed me to have if I wake up psychotic one day.

University of Manchester

Department of Psychiatry

Withington Hospital, Manchester

\section{New Publication}

MIND has published a new wall chart, Keeping It Local, which demonstrates how existing staff in health, social and community services can work together to provide the highest standard of care for all people including those who are most severely damaged through mental health problems. The chart is a guide rather than a blueprint and illus- trates how the principles MIND promotes can be put into action.

Keeping $I t$ Local is available at $£ 1.20$ (including postage) from: MIND Mail Order, 4th Floor, 24-32 Stephenson Way, London NW1 2HD. 